\title{
¿COMO ENTIENDEN LOS CONSUMIDORES LA PRODUCCIÓN Y CONSUMO SOSTENIBLES DE ALIMENTOS? UN ESTUDIO PRELIMINAR
}

\author{
$\underline{\text { Andrés Horrillo }}^{\mathrm{a}^{*}}$, Jose. A. Fernández ${ }^{\mathrm{a}}$, Alfredo. J. Escribano ${ }^{\mathrm{b}}$ Francisco. J. Mesías $^{\mathrm{a}}$ \\ a Escuela de Ingenierías Agrarias. Universidad de Extremadura - Ctra. Cáceres s/n; 06071 - Badajoz \\ (España, andreshg@unex.es;fjmesias@unex.es). ${ }^{b}$ Consultor independiente - 10005 Cáceres \\ (ajescc@gmail.com)
}

\begin{abstract}
Resumen
La creciente preocupación social por los impactos ambientales causados por la necesidad de producir alimentos y el incremento de la población mundial (Florindo et al. 2017) han hecho que aspectos como la sostenibilidad sean un tema emergente de la sociedad actual (Briggeman and Lusk 2011). Sin embargo, el concepto de sostenibilidad y lo que ésta representa, aún no están muy interiorizados entre la población. Dadas estas limitaciones, este estudio pretende medir el grado de conocimiento que tienen los consumidores sobre el concepto de sostenibilidad relacionada con el consumo y compra alimentos. Para esto, se ha utilizado un cuestionario en el que se incluyeron enunciados relativos a la sostenibilidad que los consumidores debían valorar mediante una escala Likert de 5 puntos, junto con cuestiones acerca del estilo de vida y aspectos sociodemográficos. Se obtuvieron 162 respuestas que muestran grupos significativamente diferenciados por estilos de vida. El grupo 1, dan las mayores puntuaciones al reciclaje y el medio ambiente, el grupo 2 destaca en aquellas cuestiones de salud y la alimentación y el grupo 3 dan mayor importancia al bajo precio de los alimentos.
\end{abstract}

Palabras clave: percepciones del consumidor; sostenibilidad; alimentos; investigación cualitativa; técnicas proyectivas

\section{Introducción}

La sostenibilidad es un concepto que, en la actualidad, podría decirse que está "de moda". De hecho, una breve revisión nos permite encontrar referencias a aspectos como la producción sostenible (Escribano et al. 2020), el desarrollo sostenible (Abreu et al. 2019) el consumo sostenible (Song et al. 2019), o incluso, los préstamos o la iluminación sostenibles. Esto da una idea de lo complejo que resulta definir la sostenibilidad, y por añadidura, que lo entiendan los ciudadanos. El consumo de alimentos es una de las áreas que más influye en la sostenibilidad ambiental, aunque muchos consumidores no son completamente conscientes de la asociación entre su consumo y el impacto ambiental de la producción de alimentos (Eldesouky et al. 2020). Por ello, los cambios hacia pautas de consumo más sostenibles, junto con las innovaciones tecnológicas, deberían formar parte de la solución del problema de la sostenibilidad. Por otro lado, la creciente preocupación social por los impactos ambientales causados por la necesidad de producir alimentos para satisfacer la demanda mundial (Florindo et al. 2017) ha hecho que el interés de los consumidores por la forma en que se producen sus alimentos y los métodos de producción utilizados sea cada vez mayor (Briggeman and Lusk 2011).

Esta tendencia ha llevado al crecimiento de una serie de planes de etiquetado de sostenibilidad en la industria alimentaria (Caputo et al. 2013), con los que se pretende comunicar a los consumidores información relacionada con la sostenibilidad de los alimentos. Sin embargo, la falta de familiaridad de los consumidores con el concepto de sostenibilidad dificulta la evaluación y comparación de los diferentes productos que se ofrecen (Kemp et al. 2010). Esto hace que las empresas alimentarias estén interesadas en descubrir cómo perciben los consumidores el concepto de sostenibilidad, con objeto de que la información proporcionada en acciones de promoción o en el etiquetado sea apreciada y capaz de influir en el comportamiento de compra del consumidor. En este estudio se consideró que los estilos de vida, entre los que se encuentran aspectos relacionados con la preocupación por el medio ambiente o por la salud o la adopción de formas de vida saludables (Verain et al. 2012), podían ser más fácilmente valorados por los consumidores, permitiendo así diferenciar entre grupos de consumidores en función de sus percepciones hacia la sostenibilidad.

Dadas las limitaciones ya comentadas a las que se enfrenta el estudio de la percepción del concepto de sostenibilidad, se consideró que una aproximación válida era la aplicación de técnicas de investigación cualitativa (Vaca and Mesías 2014) y en concreto de técnicas proyectivas, dado su carácter poco estructurado y flexible y adecuado para enfrentar una situación en la que los consumidores pueden ser incapaces -o al menos tener dificultades- para expresar sus percepciones hacia un concepto poco definido y con un fuerte componente subjetivo (Mesias and Escribano 2018).

\section{Metodología}


6.1. Técnicas proyectivas

Las técnicas proyectivas se basan en el principio de que los sentimientos de los encuestados y sus deseos inconscientes pueden aflorar cuando se presentan a los individuos estímulos ambiguos o mal estructurados. Los encuestados son libres de responder a estos estímulos desde su propio punto de vista, y puesto que no hay respuestas correctas o incorrectas, se puede esperar que proyecten sus sentimientos inconscientes en sus respuestas (Donoghue 2000). Se puede concluir, por tanto, que estas técnicas intentan animar a los encuestados a proyectar sus motivaciones, creencias o actitudes hacia los temas que se están investigando. Dentro de las técnicas proyectivas, en este estudio se optó por utilizar la asociación de palabras y el free listing.

- Asociación de palabras: La aplicación de la técnica de asociación de palabras se basa en pedir a los encuestados que digan lo primero que le venga a la mente al escuchar una palabra o término, o bien, se les pide que lean una lista de palabras y que digan lo que le venga a la mente con respecto a dicha lista (Mesias and Escribano 2018). Es considerada como una de las técnicas más eficaces en la investigación de mercados y es bastante frecuente en el estudio del consumo de alimentos (Banović et al. 2016; Martins et al. 2019). En este estudio, se prepararon dos tareas de asociación de palabras, solicitando para ello a los encuestados indicaran las ideas o conceptos que les venían a la mente al oír el término "sostenibilidad" (1" tarea) y "producción sostenible de alimentos" ( $2^{\mathrm{a}}$ tarea).

- Free listing: El free listing es una técnica cualitativa en la que se pide a los participantes que enumeren tantos artículos o ideas como sea posible en relación con un determinado tema (Carrillo et al. 2014). Este enfoque permite que se puedan obtener datos -en concreto, conceptos o frases- que se refieren a un único campo conceptual (Bernard 2006). El free listing puede ser usado para entender los dominios culturales y cognitivos de los individuos y para obtener una visión en las actitudes de los consumidores, especialmente cuando se enfrentan a un entorno novedoso, como puede ser el de la producción y el consumo sostenibles (Elghannam and Mesías 2018). Aunque es una herramienta simple, su potencial y fácil administración ha hecho que se convierta en una técnica cualitativa ampliamente utilizada (Morizet et al. 2011). La aplicación del free listing en esta investigación se llevó a cabo a través de la pregunta "Indique los alimentos que conoce cuya producción sea sostenible"

6.2. Recogida de datos

Se realizo mediante un cuestionario mediante la aplicación de "Google Forms", distribuido de OctubreNoviembre de 2020. Este tipo de herramientas online, dadas su flexibilidad, coste reducido y rapidez en la recogida de la información son cada vez más utilizadas en investigación. El cuestionario incluía una sección inicial con las tareas proyectivas descritas anteriormente, seguido de una serie de cuestiones acerca de los estilos de vida y aspectos sociodemográficos de los participantes. La muestra final consistió en 162 respuestas de consumidores (59,4\% mujeres y 40,6\% hombres; edad media 39,6 años). El número de encuestas final está en línea con otros estudios cualitativos (Vaca and Mesías 2014; Elghannam and Mesías 2018), considerándose además ajustada a la naturaleza introductoria de este trabajo.

6.3. Análisis de datos y segmentación

El análisis de datos se realizó de forma análoga para las tareas de asociación de palabras y free listing. Una vez agrupadas las respuestas de cada tarea, se buscaron inicialmente aquellas expresiones o términos con significados similares, que se reunieron en categorías. Finalmente, se procedió a determinar la frecuencia de mención para las categorías finales, para lo que se contó el número de participantes que habían mencionado los diferentes términos incluidos al llevar a cabo las tareas correspondientes.

A continuación, se utilizó un análisis de conglomerados para identificar subgrupos homogéneos de consumidores que pudieran revelar diferentes percepciones hacia la sostenibilidad, el cual permite un análisis más en profundidad. Los cálculos se realizaron mediante el módulo Cluster del paquete estadístico IBM SPSS 21, utilizando el procedimiento k-means. Se preguntó por los estilos de vida de los encuestados (hacer ejercicio; ahorrar energía y agua; consumo de sal; reciclaje; comer fuera de casa; comer y cocinar en casa; probar alimentos nuevos; comprobar mi salud periódicamente; consumo carne roja; comprar productos con envases reciclados; preocupación por el impacto de los alimentos; consumo fruta y verduras; etiquetado de los alimentos; intento caminar o ir en bicicleta; me preocupa el procesado de alimentos), ya que se pensó que, aunque al consumidor le resulte difícil definir su percepción de la sostenibilidad, hay hábitos de vida y consumo (sus estilos de vida) que pueden reflejar su punto de vista "sostenible" y con estos valores se aplicó un cluster k-means. Como resultado se seleccionó una solución con tres grupos debido al tamaño de los segmentos y a su importancia estadística. Un análisis de la varianza mostró que todos los segmentos diferían significativamente $(\mathrm{p}<0,001)$ entre sí con respecto a las variables incluidas en el análisis, lo que confirmó la validez de los resultados.

\section{Resultados}


Una vez definidos los grupos, los resultados del análisis de las técnicas proyectivas se muestran divididos para cada uno de los mismos en la tabla siguiente.

Cuadro 1. Frecuencias de mención por clúster (\%) para la pregunta "Indique qué ideas/conceptos le vienen a la mente al oír el término Sostenibilidad", "Indique qué ideas/conceptos le vienen a la mente al oír el término producción y consumo sostenibles de alimentos" e "Indique los alimentos de producción sostenible que conozca”.

\begin{tabular}{|c|c|c|c|c|}
\hline \multicolumn{5}{|c|}{ Indique qué ideas/conceptos le vienen a la mente al oír el término Sostenibilidad (\%) } \\
\hline Categoría & Subcategoría/Comentario & Cluster 1 & Cluster 2 & Cluster 3 \\
\hline \multirow{5}{*}{ Medio Ambiente } & Medio Ambiente & 20.65 & 25.93 & 37.25 \\
\hline & Huella de carbono & 5.16 & 1.23 & 3.92 \\
\hline & Reciclaje & 5.16 & 4.94 & 9.80 \\
\hline & Planeta & & & 1.96 \\
\hline & Energía renovable & 1.75 & & 3.92 \\
\hline \multirow{6}{*}{ Equidad } & Equilibrio & 10.33 & 7.41 & 15.69 \\
\hline & Futuro & 13.74 & 7.41 & 7.84 \\
\hline & Supervivencia & & & 1.96 \\
\hline & Mantener. conservar & & 1.23 & \\
\hline & Ética & & 1.23 & \\
\hline & Erradicar hambre & & & 1.96 \\
\hline \multirow{5}{*}{ Ecológico y natural } & Ecológico & 13.84 & 11.11 & 5.88 \\
\hline & Productos naturales. autóctonos & 5.16 & 4.94 & \\
\hline & Satisfacer necesidades humanas & & 1.23 & \\
\hline & Beneficios para la salud & & & 3.92 \\
\hline & Calidad & & 1.23 & \\
\hline \multirow{4}{*}{ Responsabilidad } & Responsabilidad & 3.41 & 8.64 & \\
\hline & Optimización de recursos & & 2.47 & \\
\hline & Consumo responsable & 3.41 & 3.70 & 1.96 \\
\hline & Autogestión & 5.26 & 2.47 & \\
\hline \multirow{4}{*}{ Economía } & Ahorro & 6.82 & & \\
\hline & Economía & 3.51 & 1.23 & \\
\hline & Economía local y social & & 7.40 & \\
\hline & Economía circular & 1.75 & 3.70 & \\
\hline
\end{tabular}

\begin{tabular}{|c|c|c|c|c|}
\hline \multicolumn{5}{|c|}{ Indique qué ideas/conceptos le vienen a la mente al oír el término producción y consumo sostenibles de alimentos (\%) } \\
\hline Categoría & Subcategoría/Comentario & Cluster 1 & Cluster 2 & Cluster 3 \\
\hline $\begin{array}{l}\text { Producción respetuosa con el } \\
\text { medio ambiente }\end{array}$ & $\begin{array}{c}\text { Producir sin comprometer el medio ambiente } \\
\text { Producir sin efectos negativos en recursos utilizados }\end{array}$ & $\begin{array}{c}36.36 \\
6.06 \\
\end{array}$ & 45.61 & $\begin{array}{c}31.58 \\
5.26 \\
\end{array}$ \\
\hline $\begin{array}{l}\text { Producción/consumo eficiente y } \\
\text { responsable }\end{array}$ & $\begin{array}{c}\text { Producir solo lo necesario } \\
\text { Máximo aprovechamiento con mínima } \\
\text { contaminación } \\
\text { Buena gestión de recursos } \\
\text { Compra eficiente evitando desperdicios } \\
\text { Producir sin consumos energéticos } \\
\text { Respeto animal } \\
\end{array}$ & $\begin{array}{r}6.06 \\
12.12 \\
15.15 \\
6.06 \\
\end{array}$ & $\begin{array}{l}14.04 \\
3.51 \\
3.51 \\
3.51 \\
\end{array}$ & $\begin{array}{c}21.05 \\
5.26 \\
13.16 \\
2.63\end{array}$ \\
\hline $\begin{array}{l}\text { Alimentación saludable y } \\
\text { natural }\end{array}$ & $\begin{array}{c}\text { Aprovechamiento de recursos cercanos. de } \\
\text { temporada } \\
\text { Producción ecológica. natural } \\
\text { Alimentación equilibrada } \\
\text { Producir mayor cantidad de alimentos saludables }\end{array}$ & 3.03 & 14.04 & 13.16 \\
\hline Equidad & $\begin{array}{c}\text { Producir sin comprometer a las generaciones futuras } \\
\text { Precio justo } \\
\text { Evitar la desnutrición mundial }\end{array}$ & $\begin{array}{l}9.09 \\
3.03\end{array}$ & $\begin{array}{l}1.75 \\
1.75\end{array}$ & $\begin{array}{l}5.26 \\
2.63 \\
\end{array}$ \\
\hline \multicolumn{5}{|c|}{ Indique los alimentos de producción sostenible que conozca (\%) } \\
\hline Categoría & Subcategoría/Producto & Cluster 1 & Cluster 2 & Cluster 3 \\
\hline Frutas y Verduras & $\begin{array}{c}\text { Frutas y verduras ecológicas } \\
\text { Uvas } \\
\text { Cerezas } \\
\text { Higos } \\
\end{array}$ & $\begin{array}{c}26.32 \\
5.26\end{array}$ & 46.15 & $\begin{array}{l}46.15 \\
7.69 \\
7.69\end{array}$ \\
\hline Alimentación general & $\begin{array}{c}\text { Café } \\
\text { Miel } \\
\text { Aceite } \\
\text { Arroz }\end{array}$ & $\begin{array}{r}5.26 \\
10.53\end{array}$ & $\begin{array}{l}3.85 \\
7.69 \\
3.85\end{array}$ & \\
\hline Ovoproductos & Huevos ecológicos & 21.05 & 11.54 & 15.38 \\
\hline Aperitivos & $\begin{array}{l}\text { Frutos secos } \\
\text { Aceitunas }\end{array}$ & $\begin{array}{l}5.26 \\
5.26 \\
\end{array}$ & 3.85 & 15.38 \\
\hline $\begin{array}{l}\text { Carne y productos cárnicos } \\
\text { procesados }\end{array}$ & $\begin{array}{l}\text { Cordero } \\
\text { Cerdo } \\
\text { Vacuno } \\
\text { Embutidos } \\
\end{array}$ & $\begin{array}{l}5.26 \\
5.26 \\
\end{array}$ & $\begin{array}{l}3.85 \\
7.69 \\
3.85\end{array}$ & \\
\hline Productos lácteos & $\begin{array}{l}\text { Leche } \\
\text { Queso }\end{array}$ & 5.26 & 3.85 & 7.69 \\
\hline
\end{tabular}




\section{Conclusiones}

Como conclusión se puede indicar que la utilización de la investigación cualitativa, y en concreto de las técnicas proyectivas, han permitido el estudio de las percepciones de los consumidores españoles hacia un concepto tan complejo y difuso como es la sostenibilidad. De entre los tres pilares tradicionales de la sostenibilidad (social, económico y ambiental) se ha podido apreciar como el componente ambiental es el que se los consumidores identifican con más facilidad, siendo los aspectos económicos los menos comentados. Cuando se intenta relacionar la sostenibilidad con la producción y el consumo de alimentos, el medio ambiente es la categoría más mencionada. Es también destacable que, a pesar del relevante papel que juega la salud en la elección de alimentos, no se ha observado una asociación entre este concepto y la sostenibilidad, incluso aunque los alimentos de producción sostenible suelen proceder de sistemas productivos menos intensivos y tradicionales, y que por ello podrían ser considerados como más saludables por los consumidores.

Otro resultado que se ha encontrado tanto en las tareas de asociación de palabras como especialmente en la de free listing, es la clara asociación entre sostenibilidad y producción ecológica, que puede llegarse a asumir que son términos intercambiables para los consumidores, lo que puede afianzar la demanda hacia este tipo de productos y ampliar su base de consumidores con aquellos conscientes de la necesidad de sistemas de producción de alimentos sostenibles.

No obstante, estas oportunidades para los productores agroalimentarios que se derivan de la mayor demanda de alimentos sostenibles chocan con la falta de familiaridad de los consumidores con el concepto, lo que les complica la evaluación y comparación de los diferentes productos que se ofrecen y puede generar confusión en el consumidor. Se hace por ello necesario educar e informar a los consumidores en el concepto de sostenibilidad, de forma que la información proporcionada por los productores sea valorada y pueda influir en el comportamiento de compra del consumidor.

\section{Bibliografía}

Abreu I, Nunes JM, Mesias FJ (2019) Can Rural Development Be Measured? Design and Application of a Synthetic Index to Portuguese Municipalities. Soc Indic Res 145:1107-1123. https://doi.org/10.1007/s11205-019-02124-w

Banović M, Krystallis A, Guerrero L, Reinders MJ (2016) Consumers as co-creators of new product ideas: An application of projective and creative research techniques. Food Res Int 87:211-223. https://doi.org/10.1016/j.foodres.2016.07.010

Bernard HR (2006) Research methods in Anthropology. Qualitative and quantitative approaches, 4th Editio. Altamira Press

Briggeman BC, Lusk JL (2011) Preferences for fairness and equity in the food system. Eur Rev Agric Econ 38:1-29. https://doi.org/10.1093/erae/jbq033

Caputo V, Nayga RM, Scarpa R (2013) Food miles or carbon emissions? Exploring labelling preference for food transport footprint with a stated choice study. Aust J Agric Resour Econ 57:465-482. https://doi.org/10.1111/1467-8489.12014

Carrillo E, Fiszman S, Lähteenmäki L, Varela P (2014) Consumers' perception of symbols and health claims as health-related label messages. A cross-cultural study. Food Res Int 62:653-661. https://doi.org/10.1016/j.foodres.2014.04.028

Donoghue S (2000) Projective techniques in consumer research. J Fam Ecol Consum Sci 28:47-53. https://doi.org/10.4314/jfecs.v28i1.52784

Eldesouky A, Mesias FJ, Escribano M (2020) Consumer assessment of sustainability traits in meat production. A choice experiment study in Spain. Sustainability 12:4093. https://doi.org/10.3390/su12104093

Elghannam A, Mesías FJ (2018) Social networks as a new marketing channel for animal food products: A qualitative study in Spain. Arch Zootec 67:. https://doi.org/10.21071/az.v67i258.3662

Escribano M, Gaspar P, Mesias FJ (2020) Creating market opportunities in rural areas through the development of a brand that conveys sustainable and environmental values. J Rural Stud 75:206215. https://doi.org/10.1016/j.jrurstud.2020.02.002

Florindo TJ, de Medeiros Florindo GIB, Talamini E, et al (2017) Carbon footprint and Life Cycle Costing of beef cattle in the Brazilian midwest. J Clean Prod 147:119-129. https://doi.org/10.1016/j.jclepro.2017.01.021

Kemp K, Insch A, Holdsworth DK, Knight JG (2010) Food miles: Do UK consumers actually care? Food Policy 35:504-513. https://doi.org/10.1016/j.foodpol.2010.05.011

Martins IBA, Oliveira D, Rosenthal A, et al (2019) Brazilian consumer's perception of food processing technologies: A case study with fruit juice. Food Res Int 125:108555. https://doi.org/10.1016/j.foodres.2019.108555

Mesias FJ, Escribano M (2018) Projective techniques. In: Ares G, Varela P (eds) Methods in Consumer 
Research, Volume 1. New approaches to classic methods. Elsevier Ltd, pp 79-102

Morizet D, Depezay L, Masse P, et al (2011) Perceptual and lexical knowledge of vegetables in preadolescent children. Appetite 57:142-147. https://doi.org/10.1016/j.appet.2011.04.006

Song L, Lim Y, Chang P, et al (2019) Ecolabel's role in informing sustainable consumption: A naturalistic decision making study using eye tracking glasses. J Clean Prod 218:685-695. https://doi.org/10.1016/j.jclepro.2019.01.283

Vaca SI, Mesías FJ (2014) Percepciones de los consumidores españoles hacia las frutas de Ecuador: Un estudio preliminar cualitativo con técnicas proyectivas. ITEA Inf Tec Econ Agrar 110:89-101. https://doi.org/10.12706/itea.2014.006

Verain MCD, Bartels J, Dagevos H, et al (2012) Segments of sustainable food consumers: a literature review. Int J Consum Stud 36:123-132. https://doi.org/10.1111/j.1470-6431.2011.01082.x 